\title{
Ocorrência de Rhinonyssus rhinolethrum (Trouessart, 1895) (Acarina: Rhinonyssidae) em marreca caneleira Dendrocygna bicolor (Vieillot, 1816) e marreca piadeira Dendrocygna viduata (Linnaeus, 1766) na região sul do Rio Grande do Sul, Brasil*
}

\author{
Occurrence of Rhinonyssus rhinolethrum (Trouessart, 1895) \\ (Acarina: Rhinonyssidae), a nasal mite in fulvous whistling duck Dendrocygna \\ bicolor (Vieillot, 1816) and white-faced whistling-duck Dendrocygna \\ viduata (Linnaeus, 1766) in Southern Brazil
}

\author{
Afonso Lodovico Sinkoc ${ }^{1 * *}$, João Guilherme Werner Brum², Gertrud Muller²

RESUMO: Os autores relatam a ocorrência do ácaro nasal Rhinonyssus rhinolethrum (Trouessart, 1895) (Acarina: Rhinonyssidae) em marreca caneleira Dendrocygna bicolor (Vieillot, 1816) e marreca piadeira Dendrocygna viduata (Linnaeus, 1766) de vida livre na regiáo sul do Rio Grande do Sul. Para a prevalência e intensidade média foram observados $35,5 \%$ e 2,27 ácaros para $D$. bicolor e $13 \%$ e 9,33 ácaros para $D$. viduatta. A intensidade de parasitismo observada variou de 1 a 6 ácaros em $D$. bicolor e 1 a 22 ácaros em D. viduatta. O parasitismo caracteriza o primeiro relato da ocorrência de $R$. rhinolethrum sobre Dendrocygna bicolor e sobre Dendrocygna viduata no Rio Grande do Sul, ampliando a listagem dos anatídeos parasitados por essa espécie neste Estado.

PALAVRAS-CHAVE: marreca caneleira; Dendrocygna bicolor; ácaro nasal; Rhinonyssidae; marreca piadeira; Dendrocygna viduata.

\begin{abstract}
The authors report the occurrence of the nasal mite Rhinonyssus rhinolethrum (Trouessart, 1895) (Acarina: Rhinonyssidae) in free-living fulvous whistling duck Dendrocygna bicolor (Vieillot, 1816) and white-faced whistling-duck Dendrocygna viduata (Linnaeus, 1766) in the Rio Grande do Sul region, Southern Brazil. Prevalence and mean intensity showed values of $35.5 \%$ and 2.27 mites to $D$. bicolor and $13 \%$ and 9.33 mites for D. viduatta. The intensity of parasitism ranged from 1 to 6 mites in D. bicolor, and 1 to 22 mites in D. viduatta. This is the first report of $R$. rhinolethrum on D. bicolor and D. viduata in Rio Grande do Sul, expanding the list of anatids parasitized by this species in this State.
\end{abstract}

KEYWORDS: fulvous whistling duck; Dendrocygna bicolor; nasal mite; Rhynonyssidae; white-faced whistling-duck; Dendrocygna viduata.

'Faculdade de Agronomia, Medicina Veterinária e Zootecnia, Departamento de Clínica Médica Veterinária, Universidade Federal de Mato Grosso (UFMT) - Cuiabá (MT), Brasil. ${ }^{2}$ Departamento de Microbiologia e Parasitologia, Instituto de Biologia, Universidade Federal de Pelotas (UFPel) - Pelotas (RS), Brasil.

*Este artigo faz parte da tese de doutoramento do primeiro autor.

**Autor correspondente: alsinkoc@gmail.com

Recebido em: 12/09/2014. Aceito em: 20/02/2016 
O Rio Grande do Sul alberga 21 das 25 espécies dos Anatidae (Anseriformes) encontrados no Brasil (Belton, 1994), dentre elas Dendrocygna bicolor (Vieillot, 1816) e D. viduata (Linnaeus, 1766), que são residentes e abundantes no estado (SICK, 1997).

Ácaros que são encontrados nas vias nasais de aves Anatidae pertencem às famílias Ereynethidae e Rhinonyssidae, destacando-se duas espécies: Ophthalmognathus womersleyi (Fain, 1955) e Rhinonyssus rhinolethrum (Trouessart, 1895), respectivamente, sendo a segunda também encontrada em uma espécie de Rallidae (Gruiformes) (Mitchell; Rhodes, 1960; Mitchel, 1963; Pence, 1975).

Nos Estados Unidos, Mitchell; Rhodes (1960) encontraram 12 espécies de anatídeos parasitados por $R$. rhinolethrum, sendo que apenas para três delas, Anser caerulescens (Linnaeus, 1758), A. hyperborea (Linnaeus, 1758) e Anasdiscors (Linnaeus, 1766), há dados epizootiológicos com prevalência $(40,00$; 32,35 e $21,87 \%$, respectivamente) e intensidade média de parasitismo $(6,16 ; 5,36$ e 12,86, respectivamente).

Pence (1972) encontrou $R$. rhinolethrum em duas espécies de Anatidae e em uma espécie de Rallidae (Fulica americana Gmelin, 1789). Trabalhando com ácaros nasais dessas aves na América do Norte, Pence (1975) citou 15 espécies de anatídeos e a mesma de Rallidae como hospedeiras para $R$. rhinolethrum; enquanto que Ophthalmognathus womersleyi foi encontrado apenas em três espécies desses anatídeos.

SPICER (1987) relatou a ocorrência de $R$. rhinolethrum com prevalência de $20 \%$ em Aythyaaffinis (Eyton, 1838) e Ophthalmognathus womersleyi com $50 \%$ em Anas clypeata (Linnaeus, 1758).

George; Bolen (1975) relataram no Texas (EUA) a presença de $R$. rhinolethrum sobre Dendrocygna autumnalis (Linnaeus, 1758) com 13\% de prevalência e intensidade média de parasitismo de três ácaros, sendo observado o parasitismo de um a sete ácaros por ave.

No Brasil, $R$. rhinolethrum foi observado sobre $D$. viduatta na Fundação Parque Zoológico de São Paulo (Amaral, 1968) e em Santo Amaro e Cananeia, São Paulo (Amaral; Rebouças, 1974).

No estado do Rio Grande do Sul, $R$. rhinolethrum foi relatado parasitando Netta peposaca (Paulsen, 2006), Callonetta leucophrys e Cygnus melanocoryphus (MasCarenhas et al. 2009).

Neste estudo, foram analisados 31 indivíduos de D. bicolor e 23 de D. viduata; as aves foram coletadas em 2005 nos municípios de Pelotas e Arroio Grande, Rio Grande do Sul, por meio da caça com arma de fogo sob a licença número 053/2005-DITEC/IBAMA-RS, Protocolo IBAMA $\mathrm{n}^{\circ}$ 02023.002619/03-14. Após o abate, as aves foram acondicionadas em sacos plásticos individuais e refrigeradas até a chegada ao laboratório, onde a cavidade nasal foi dissecada e lavada sob jato de água. O conteúdo da lavagem foi transferido para um balde, posteriormente tamisado (malha $0,15 \mathrm{~mm}$ ) e inspecionado ao estereomicroscópio à procura de parasitos.
Os ácaros encontrados foram fixados e conservados em álcool $70^{\circ} \mathrm{GL}$. Os ácaros foram clarificados em líquido de Nesbitt, montados entre lâmina e lamínula em meio de Hoyer's e examinados ao microscópio ótico para identificação específica. Eles foram identificados de acordo com Castro (1948), Mitchell (1963), Pence (1975) e Krantz (1978).

Os parâmetros prevalência, intensidade média, abundância média e intensidade parasitária foram definidos de acordo com Bush et al. (1997) e analisados por meio do software Quantitative Parasitology 3.0 (Rózsa et al., 2000).

Foi identificado um total de 53 ácaros pertencentes a Rhinonyssus rhinolethrum (Trouessart, 1895), parasitando D. viduata e D. bicolor (Tabela 1 ).

O diagnóstico do parasitismo de aves por ácaros nasais é extremamente dependente do número de indivíduos examinados. Segundo SPICER (1987), a probabilidade aumenta uma vez que um número mínimo de dez hospedeiros é analisado. Como a filogenia dos hospedeiros pode afetar a habilidade do ácaro em parasitar as vias nasais de seus hospedeiros, a prevalência parece diferir significativamente entre regiôes geográficas e as espécies de hospedeiros examinadas (SPICER, 1987).

Embora $R$. rhinolethrum tenha sido encontrado em $D$. viduata e $D$. bicolor, e sabe-se que esse ácaro possui uma ampla distribuição geográfica e é encontrado em muitas espécies de Anatidae (MrTcheL, 1963), no Brasil havia apenas sido observado em D. viduata (AmARAL, I968; Amaral; RebouçAS, 1974), após relatos já configurados em outras espécies do gênero Dendrocygna (Swainson, 1837) (George; Bolen, 1975). Os estudos de prevalência de ácaros Rhinonyssidae em aves têm demonstrado valores variando de 16 a 24\% (Pence, 1972; Spicer, 1984, 1987). Em Anatidae, relatos de prevalência são conhecidos para Anser caerulescens (40\%), A. hyperborea (32,35\%), Anas discors (21,87\%) (MrtCHeLL; RHODES, 1960) e D. autumnalis (13\%) (GeORGE; Bolen, 1975), sendo próximos dos valores encontrados para $D$. viduata $(13 \%)$ e $D$. bicolor (35,5\%) no Rio Grande do Sul.

Poucos dados comparativos são disponibilizados sobre a intensidade média e de parasitismo por $R$. rhinolethrum. Em D. bicolor, a intensidade média de 2,27 ácaros foi inferior àquelas citadas para $D$. autumnalis de três ácaros (GeOrge; Bolen, 1975) e para A. caerulescens de 6,16 ácaros, para $A$. hyperborea de 5,36 ácaros e para Anas discors de 12,86 ácaros (Mitchell;

Tabela 1. Prevalência, intensidade média, abundância média e intensidade de parasitismo de Rhinonyssus rhinolethrum em Dendrocygna bicolor e $D$. viduata na região sul do Rio Grande do Sul, capturadas em 2005.

\begin{tabular}{lcccc} 
Espécies & $\begin{array}{c}\text { Prevalência Intensidade } \\
\text { (\%) }\end{array}$ & $\begin{array}{c}\text { Abundância } \\
\text { média }\end{array}$ & $\begin{array}{c}\text { Intensi- } \\
\text { média }\end{array}$ & dade \\
\hline $\begin{array}{l}\text { D. bicolor } \\
(n=31)\end{array}$ & 35,5 & 2,27 & 0,81 & $1-6$ \\
\hline $\begin{array}{l}\text { D. viduata } \\
(n=23)\end{array}$ & 13,0 & 9,33 & 1,22 & $1-22$ \\
\hline
\end{tabular}


RHODEs, 1960). Embora com níveis de prevalência mais baixa, D. viduata apresentou maior intensidade média, sendo que, em um indivíduo, notou-se a maior intensidade de parasitismo, 22 ácaros coletados, totalizando $41,51 \%$ do total deles.

Os meios de transmissão de ácaros Rhinonyssidae são desconhecidos, entretanto, StRANDMANn (1951) considerou que essa espécie seja transmitida, provavelmente, durante a atividade de preening (do tipo allopreening); possivelmente a densidade e os fatores comportamentais intra e interespecíficos dos hospedeiros afetem a distribuição de $R$. rhinolethrum entre populaçóes de anatídeos na área estudada.
Este é o primeiro relato da ocorrência de Rhinonyssus rhinolethrum sobre Dendrocygna bicolor e D. viduata no Rio Grande do Sul, ampliando a listagem dos anatídeos parasitados por essa espécie no estado.

\section{AGRADECIMENTOS}

Agradecemos a M. M. Rebouças pelo envio de material bibliográfico e a M. P. Valim pelas sugestôes na revisão do manuscrito.
AMARAL, V. Notas sobre ácaros nasais com a descrição de duas novas espécies: Ptilonyssus zeferinoi in. sp. e Sternostoma clementei n. sp. (Acarina: Rhinonyssidae). Lista das espécies descritas no Brasil e seus hospedeiros. Arquivos do Instituto Biológico, v.35, n.3, p.107-126, 1968.

AMARAL, V.; REBOUÇAS, M.M. Notas sobre ácaros rinonissideos de aves brasileiras (Mesotigmata-Rhinonyssidae). O Biológico, v.40, p.52-56, 1974

BELTON, W. Aves do Rio Grande do Sul: distribuição e biologia. Tradução de Teresinha Tesche Roberts. São Leopoldo: Unisinos, 1994. 584p.

BUSH, A.O.; LAFFERTY, K.D.; LOTZ, J.M.; SHOSTAK, A.W. Parasitology meets ecology on its own terms: Margolis et al. revisited. The Journal of Parasitology, v.83, n.4, p.575-583, 1997.

CASTRO, M.P. Reestruturação genérica da familia Rhinonyssidae Vitzthum, 1935 (Acari: Mesostigmata: Gamasides) e descrição de algumas espécies novas. Arquivos do Instituto Biológico, v. 18 , p.253-284, 1948.

GEORGE, R.R.; BOLEN, E.G. Endoparasites of black-bellied whistling ducks in southern Texas. Journal of Wildlife Diseases, v.11, n.1, p.11-22, 1975.

KRANTZ, G.W. A manual of acarology. 2 ed. Corvallis: OSU Beaver Store, 1978. 509p.

MASCARENHAS, C.S.; BRUM, J.G.W.; COIMBRA, M.A.A.; SINKOC, A.L. Novos hospedeiros para o ácaro nasal Rhinonyssus rhinolethrum (Trouessart) (Gamasida: Rhinonyssidae) no Brasil. Neotropical Entomology, v.38, n.5, p.695-696, 2009.

MITCHELL, R.W. Comparative morphology of the life stages of the nasal mite Rhinonyssus rhinolethrum (Mesostigmata: Rhinonyssidae). The Journal of Parasitology, v.49, n.3, p.506-515, 1963.
MITCHELL, R.W.; RHODES, W.L. New host records for the mesostigmatid nasal mite Rhinonyssus rhinolethrum (Acarina: Rhinonyssidae). Southwestern Naturalist, v.5, n.2, p.107-108, 1960.

PAULSEN, R.M.M. Caracterização de helmintos e artrópodes parasitos de Netta peposaca (Vieillot, 1816) (Marrecão-dapatagônia) (Aves: Anatidae) no Rio Grande do Sul. 2006. 100p. Tese (Doutorado em Ciências Veterinárias) - Faculdade de Veterinária, Universidade Federal do Rio Grande do Sul, Porto Alegre, 2006.

PENCE, D.B. The nasal mites of birds from Louisiana. I. Dermanyssids (Rhinonyssinae) from shore and marsh birds. The Journal of Parasitology, v.58, n.1, p.153-168, 1972.

Keys, species and host list, and bioliography for nasal mites of North American birds (Acarina: Rhinonyssinae, Turbinoptinae, Speleognathinae, and Cytoditidae). Texas: Special Publications the Museum Texas Tech University, 1975, 148p.

RÓZSA, L.; REICZIGEL, J.; MAJOROS, G. Quantifying parasites in samples of hosts. The Journal of Parasitology, v.86, n.2, p.228-232, 2000.

SICK, H. Ornitologia brasileira. Rio de Janeiro: Nova Fronteira, 1997. $912 \mathrm{p}$.

SPICER, G.S. Nasal mites from birds of a Guatemalan cloud forest (Acarina: Rhinonyssidae). The Journal of Parasitology, v.70, n.5, p.794-802, 1984.

Prevalence and host-parasite list of some nasal mites from birds (Acarina: Rhinonyssidae, Speleognathidae. The Journal of Parasitology, v.73, n.2, p.259-264, 1987.

STRANDTMANN, R.W. The mesostigmatic nasal mites of birds. II. New and poorly known species of Rhinonyssidae. The Journal of Parasitology, v.37, n.2, p.129-140, 1951. 